\title{
The Modeling of Chikungunya Using Lagrange Method and Lagrange Method by Matlab
}

\author{
Abdel Radi Abdel Rahman Abdel Gadir ${ }^{1}$, Subhi Abdalazim Aljily ${ }^{2}$, Neama Yahia Mohammed ${ }^{3}$ \\ ${ }^{1}$ Department of Mathematics, Faculty of Education, Omdurman Islamic University, Omdurman, Sudan \\ ${ }^{2}$ Department of Mathematics, Faculty of Education, University of AL Butana, Rufaa, Sudan \\ ${ }^{3}$ Department of Mathematics, College of Science, Tabuk University, Tabuk, Saudi Arabia
}

Email address:

dibdelradi78@gmail.com (A. R. A. R. A. Gadir)

To cite this article:

Abdel Radi Abdel Rahman Abdel Gadir, Subhi Abdalazim Aljily, Neama Yahia Mohammed. The Modeling of Chikungunya Using Lagrange Method and Lagrange Method by Matlab. American Journal of Applied Mathematics. Special Issue: Numerical Analysis and Control Theory. Vol. 9, No. 1, 2021, pp. 1-9. doi: 10.11648/j.ajam.20210901.11

Received: July 26, 2020; Accepted: December 5, 2020; Published: January 12, 2021

\begin{abstract}
Chikungunya virus (CHIKV) is a mosquito-transmitted alphavirus that causes acute fever and acute and chronic musculoskeletal pain in humans, there is currently no vaccine, cure or specific treatment for Chikungunya. Chikungunya originated in Africa and has since spread across the entire globe causing large numbers of epidemics that have infected millions of people in Asia, Indian subcontinent, Europe, the Americas, and Pacific Islands. Adequate coordinated efforts comprising active surveillance, early detection, vector control and public awareness at local, national and international level need to be adopted in endemic areas for the effective control of Chikungunya virus infection. There is a risk that the virus will be imported to new areas by infected travelers. There is no vaccine to prevent or medicine to treat chikungunya virus infection. Travelers can protect themselves by preventing mosquito bites. The aims of this paper is to study Chikungunya virus and to illustrate the possibility of its modeling by Lagrange method using Matlab. Also we made modeling of results of tests for patients with Chikungunya numerically using Lagrange interpolating method and using Lagrange interpolating method by Matlab which is one of the most famous mathematical programs in the mathematical modeling of mathematical problems. We followed the numerical method and applied mathematical method using Matlab. We found that the modeling using Lagrange interpolating method by Matlab is more accuracy and speed than the numerical method were we explained this fact that we have reached in three figures which proves the aptitude the usage of Matlab in mathematical modeling.
\end{abstract}

Keywords: Modeling, Chikungunya, Lagrange Method, Matlab

\section{Introduction}

Chikungunya fever is a viral infection affecting all age groups [6], Chikungunya virus, or the CHIK virus in short, is an RNA virus that belongs to the alpha virus genus of the Togaviridae family, the latter comprising viruses that are mostly transmitted by arthropods. Infection with CHIK virus results in chikungunya fever. The name Chikungunya derives from a root verb in the Kimakonde language meaning "that which bends up", i.e. to become contorted. The name reflects the stooped appearance of sufferers with arthralgia [17], Mosquito-borne arboviruses like dengue (DENV), Zika (ZIKV), and Chikungunya (CHIKV) are important public health challenges in tropical and subtropical regions [10],
The disease was documented first time in the form of $n$ outbreak in Tanzania [18], The chikungunya virus (CHIKV) isanenveloped, spherical, single-stranded positive-sense RNA alpha virus belonging to the family Togaviridae [1], The virus is amember of the Semliki Forest virus antigenic complex, which contains important arboviruses such as O'nyongnyong virus (ONNV) from Africa, Mayaro virus (MAYV) from South America and Ross River virus (RRV) from the Oceania region [12] hikungunya is a vectorborne viral disease thatcauses large outbreaks, mainly in tropical and subtropical countries [9] Chikungunya fever (CF) is a viral illness caused by an arbovirus transmitted by the Aedes mosquitoes. The disease was documented first time in the form of an outbreak in Tanzania. The name is derived from the 'makonde' dialect which means 'that which bends up', 
indicating the physical appearance of a patient with severe clinical features. [15]

The general objectives of the guidelines are to assist member countries inpreventing and controlling outbreaks of chikungunya fever by surveillance, case detection, investigation, and by triggering necessary public healthactions. [7]

\section{Signs and Symptoms}

Depending on the duration of symptoms, CHIKV infection may be divided into acute(the first 3 weeks from onset), postacute (from 21st day to the end of 3rd month) and chronic stage (after 3 months from onset) [13], Depending on the duration of symptoms, CHIKV infection may be divided into acute(the first 3 weeks from onset), post-acute (from 21st day to the end of 3rd month) and chronic stage (after 3 months from onset) [5]. PROGNOSIS, OUTCOME, AND PREVENTION Several agents have demonstrated activity against CHIKVin vitro, and monoclonal antibodies have shown efficacy in animal models (17-175), but currently, there is no specific antiviral treatment for CHIKV and management remains symptomatic. Joint pain, the symptoms of acute chikungunya typically resolve over a few days to 1 week $(1,68)$. Approximately $50 \%$ of patients have chronic arthralgia and/or arthritis at 3 to 6 months, and over $25 \%$ of patients may still have symptoms at 12 months, which negatively impacts quality of life during recovery $(68,176-180)$. [8] Symptoms of chikungunya are fever and severejoint pain that can last for weeks. Other symptoms include muscle pain, headache, nausea, fatigue and rash. Most patients recover fully, but in some cases joint pain may persist for several months oreven years. Occasional cases of eye, neuro logical and heart complications have been reported, as well as gastro intestinal complaints. Serious complications are not common but, in older people, the disease can contribute to the cause of death [3].

\section{Treatment, Prevention and Indicators}

There is currently no vaccine, cure or specific treatment for Chikungunya. Patient care is generally focusedon providing comfort through relief of symptoms. Medicines for reducing fever and pain such as a cetaminophen, (commonly marketed as paramol, panadol, paracetamol, etc.,) [13], Treatment is predominantly supportive and includes bed rest during the febrile period, adequate hydration, and antipyretics and analgesics [6], In acute stage, the treatment is mainly supportive and consists of fluids, analgesics and antipyretics [5]. There have been no case reports of laboratory transmission from blood products; however, a nurse reported acquiring CHIKV by drawing the blood of an infected patient (80). As such, continued caution is recommended when handling infected blood products or live virus in the laboratory due to the potential for transmission. [8] As CHIK fever is an emerging disease it has not received sufficient coverage yetin themedical curricula. Specific treatment is not a vailable, and there is no vaccine. This publication comprises the experience of experts as published in WHO/SEAROand PAHO/WHO guidelines[14]

Rate the positives of homes, Rate of positive utensils, Rate of virginity, Preto rate [6].

\section{Clinical Findings and Diagnosis}

The clinical findings of CHIK Vinfection very much similar to those of Dengue and Zika and in areas lacking laboratory diagnostic facilities, CHIK Visprobably frequently under diagnosed or misdiagnosed as Dengueor Zika. The incubation period of CVIKV varies from 2to 12 days following the bite of infected mosquito, withan average of 2 to 7 days. The disease is characterized by suddenonse to fhigh fever and arthralgia or arthritis(Hoque and Ahmed, 2012). Several joints of the body may be affected, found swollen. The fever may persist for 8 to 10 days. [7] The percentage of subclinical infections fluctuates between epidemics and it depends on factors such as age, naïve population /herd immunity, and the circulating strain [2] The classic triad of symptomatic chikungunya includes an abruptonset febrile illness, severe and often debilitating arthralgias, and a rash [8], Many patients that progress to Subacute/Chronic phases have neuropathic pain (NP) besides the articular symptoms. The NP is associated with a less favorable outcome, with greater impact on quality of life and lower efficacy of treatment [4]

\section{CausativeAgent and Vectors}

Chikungunya fever is primarily transmitted by bites of mosquitoes ofthe genus Aedes, the same mosquito that transmits Dengue Fever. Onlythe female mosquitoes are infective because they require a blood mealfor the formation of the egg. Of the two vectors in Asia, Aedesaegyptiisbe lieved to be the principal vector responsible for transmission during human out breaks. Aedesaegypti breeds in stored freshwater in urban and semi-urban environments, The Aedes mosquitoes breed in domestic settings such as flower vases, water-storage containers, desert coolers, etc and peridomestic areas such as construction sites, coconut shells, discarded household junk items (vehicular, plastic and metal cans, etc). Adult mosquitoes rest in cool and shady areas in domestic and peridomestic settings and bite humans during the daytime [17]

\section{Polynomial Interpolation Method}

If we have $(n+1)$ points $\left\{\left(x_{i}, y_{i}\right) ; i=0,1,2, \ldots n\right\}$ then we will be able to make polynomial furcation from $(n)$ degree $\left\{p_{n}(x)\right\}$ using any one of the interpolation methods mentioned (Nyazi Tawfeeg Ahmed, 2001).

The General Formula Is Given by

$$
P_{n}(x)=\sum_{l>0}^{n} l_{i}(x) y_{i}
$$

The terms $l_{0}(x), l_{1}(x), l_{2}(x), \ldots, l_{n}(x)$ are known as 
Lagrange polynomials and they can be obtained by the following relation:

$$
l_{i}(x)=\prod_{\substack{j=0 \\ j \neq i}}^{n} \frac{x-x_{i}}{x_{i}-x_{j}} ; i=0,1,2, \ldots n
$$

(Nyazi Tawfeeg Ahmed,2001) [11] polynomial function of the five degree which interpolates the depicted table.

\section{Health Centers}

\subsection{Railway Health Center}

Table 1. A table showing that the number of those who came to Railway Health Center and who were injured

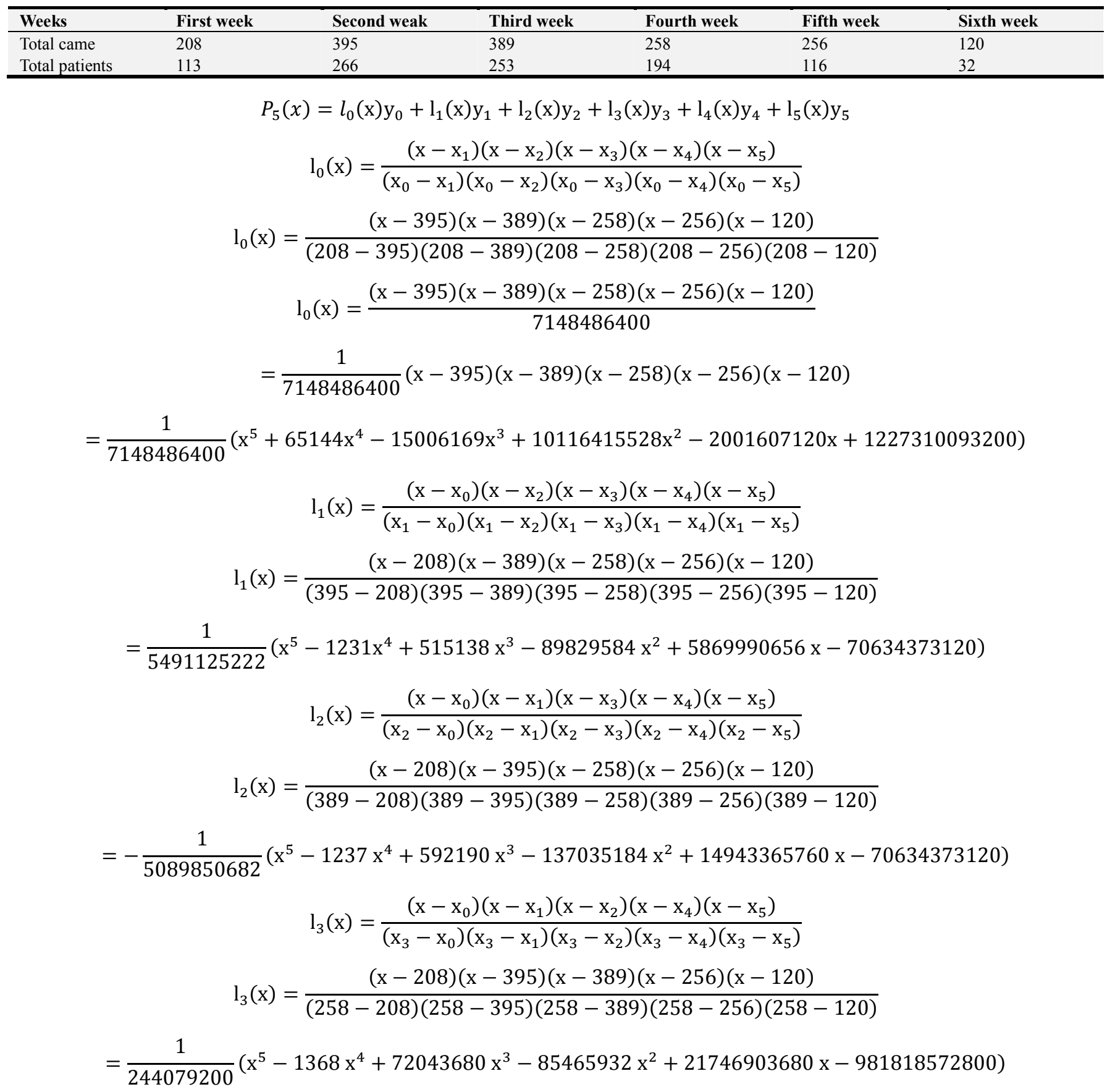




$$
\begin{aligned}
& \mathrm{l}_{4}(\mathrm{x})=\frac{\left(\mathrm{x}-\mathrm{x}_{0}\right)\left(\mathrm{x}-\mathrm{x}_{1}\right)\left(\mathrm{x}-\mathrm{x}_{2}\right)\left(\mathrm{x}-\mathrm{x}_{3}\right)\left(\mathrm{x}-\mathrm{x}_{5}\right)}{\left(\mathrm{x}_{4}-\mathrm{x}_{0}\right)\left(\mathrm{x}_{4}-\mathrm{x}_{1}\right)\left(\mathrm{x}_{4}-\mathrm{x}_{2}\right)\left(\mathrm{x}_{4}-\mathrm{x}_{3}\right)\left(\mathrm{x}_{4}-\mathrm{x}_{5}\right)} \\
& \mathrm{l}_{4}(\mathrm{x})=\frac{(\mathrm{x}-208)(\mathrm{x}-395)(\mathrm{x}-389)(\mathrm{x}-258)(\mathrm{x}-120)}{(256-208)(256-395)(256-389)(256-258)(256-120)} \\
& =-\frac{1}{241366272}\left(x^{5}-1370 x^{4}+722663 x^{3}-182395366 x^{2}+21886838640 x-989489030400\right) \\
& l_{5}(x)=\frac{\left(x-x_{0}\right)\left(x-x_{1}\right)\left(x-x_{2}\right)\left(x-x_{3}\right)\left(x-x_{4}\right)}{\left(x_{5}-x_{0}\right)\left(x_{5}-x_{1}\right)\left(x_{5}-x_{2}\right)\left(x_{5}-x_{3}\right)\left(x_{5}-x_{4}\right)} \\
& \mathrm{l}_{5}(\mathrm{x})=\frac{(\mathrm{x}-208)(\mathrm{x}-395)(\mathrm{x}-389)(\mathrm{x}-258)(\mathrm{x}-256)}{(120-208)(120-395)(120-389)(120-258)(120-256)} \\
& =-\frac{1}{122175926400}\left(x^{5}-1506 x^{4}+892663 x^{3}-491277534 x^{2}+176639748256 x-21089869931521\right) \\
& P_{5}(x)=l_{0}(\mathrm{x}) \mathrm{y}_{0}+\mathrm{l}_{1}(\mathrm{x}) \mathrm{y}_{1}+\mathrm{l}_{2}(\mathrm{x}) \mathrm{y}_{2}+\mathrm{l}_{3}(\mathrm{x}) \mathrm{y}_{3}+\mathrm{l}_{4}(\mathrm{x}) \mathrm{y}_{4}+\mathrm{l}_{5} P_{5}(x) \\
& P_{5}(x)=\left[\left(\frac{1}{7148486400}\left(x^{5}+65144 x^{4}-15006169 x^{3}+10116415528 x^{2}-2001607120 x+1227310093200\right)\right) 113\right] \\
& +\left[\left(\frac{1}{5491125222}\left(x^{5}-1231 x^{4}+515138 x^{3}-89829584 x^{2}+5869990656 x-70634373120\right)\right) 266\right] \\
& +\left[\left(-\frac{1}{5089850682}\left(x^{5}-1237 x^{4}+592190 x^{3}-137035184 x^{2}+14943365760 x-70634373120\right)\right) 253\right] \\
& +\left[\left(\frac{1}{244079200}\left(x^{5}-1368 x^{4}+72043680 x^{3}-85465932 x^{2}+21746903680 x-981818572800\right)\right) 194\right] \\
& +\left[\left(-\frac{1}{241366272}\left(x^{5}-1370 x^{4}+722663 x^{3}-182395366 x^{2}+21886838640 x-989489030400\right)\right) 116\right] \\
& +\left[\left(-\frac{1}{122175926400}\left(x^{5}-1506 x^{4}+892663 x^{3}-491277534 x^{2}+176639748256 x\right.\right.\right. \\
& -21089869931521)) 32]
\end{aligned}
$$

\subsection{Pante 42 Health Center}

Table 2. A table showing that the number of those who came to Pante 42 Health Center and who were injured

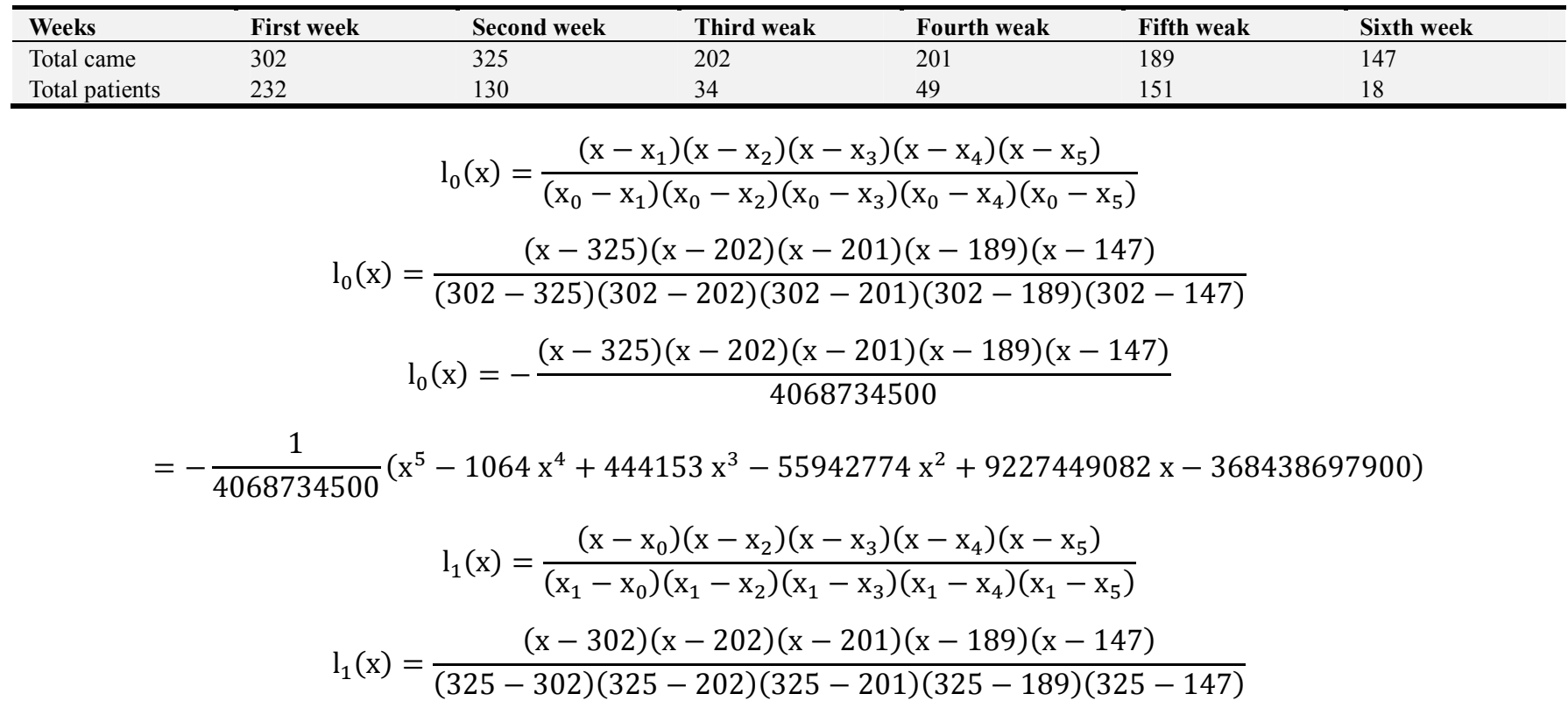




$$
\begin{aligned}
& =\frac{1}{8492069568}\left(x^{5}-1041 x^{4}+426971 x^{3}-86384307 x^{2}+8629369308 x-340669700532\right) \\
& \mathrm{l}_{2}(\mathrm{x})=\frac{\left(\mathrm{x}-\mathrm{x}_{0}\right)\left(\mathrm{x}-\mathrm{x}_{1}\right)\left(\mathrm{x}-\mathrm{x}_{3}\right)\left(\mathrm{x}-\mathrm{x}_{4}\right)\left(\mathrm{x}-\mathrm{x}_{5}\right)}{\left(\mathrm{x}_{2}-\mathrm{x}_{0}\right)\left(\mathrm{x}_{2}-\mathrm{x}_{1}\right)\left(\mathrm{x}_{2}-\mathrm{x}_{3}\right)\left(\mathrm{x}_{2}-\mathrm{x}_{4}\right)\left(\mathrm{x}_{2}-\mathrm{x}_{5}\right)} \\
& l_{2}(x)=\frac{(x-302)(x-325)(x-201)(x-189)(x-147)}{(202-302)(202-325)(202-201)(202-189)(202-147)} \\
& =\frac{1}{8794500}\left(x^{5}-1164 x^{4}+530168 x^{3}-118055946 x^{2}+12856967991 x-548107191450\right) \\
& \mathrm{l}_{3}(\mathrm{x})=\frac{\left(\mathrm{x}-\mathrm{x}_{0}\right)\left(\mathrm{x}-\mathrm{x}_{1}\right)\left(\mathrm{x}-\mathrm{x}_{2}\right)\left(\mathrm{x}-\mathrm{x}_{4}\right)\left(\mathrm{x}-\mathrm{x}_{5}\right)}{\left(\mathrm{x}_{3}-\mathrm{x}_{0}\right)\left(\mathrm{x}_{3}-\mathrm{x}_{1}\right)\left(\mathrm{x}_{3}-\mathrm{x}_{2}\right)\left(\mathrm{x}_{3}-\mathrm{x}_{4}\right)\left(\mathrm{x}_{3}-\mathrm{x}_{5}\right)} \\
& l_{3}(x)=\frac{(x-302)(x-325)(x-202)(x-189)(x-147)}{(201-302)(201-325)(201-202)(201-189)(201-147)} \\
& =-\frac{1}{8115552}\left(x^{5}-1165 x^{4}+488045 x^{3}-91377629 x^{2}+8678475432 x-550834092900\right) \\
& \mathrm{l}_{4}(\mathrm{x})=\frac{\left(\mathrm{x}-\mathrm{x}_{0}\right)\left(\mathrm{x}-\mathrm{x}_{1}\right)\left(\mathrm{x}-\mathrm{x}_{2}\right)\left(\mathrm{x}-\mathrm{x}_{3}\right)\left(\mathrm{x}-\mathrm{x}_{5}\right)}{\left(\mathrm{x}_{4}-\mathrm{x}_{0}\right)\left(\mathrm{x}_{4}-\mathrm{x}_{1}\right)\left(\mathrm{x}_{4}-\mathrm{x}_{2}\right)\left(\mathrm{x}_{4}-\mathrm{x}_{3}\right)\left(\mathrm{x}_{4}-\mathrm{x}_{5}\right)} \\
& \mathrm{l}_{4}(\mathrm{x})=\frac{(\mathrm{x}-302)(\mathrm{x}-325)(\mathrm{x}-202)(\mathrm{x}-201)(\mathrm{x}-147)}{(189-302)(189-325)(189-202)(189-201)(189-147)} \\
& =\frac{1}{100691136}\left(x^{5}-1177 x^{4}+542843 x^{3}-122552555 x^{2}+13541836188 x-585807686100\right) \\
& \mathrm{I}_{5}(\mathrm{x})=\frac{\left(\mathrm{x}-\mathrm{x}_{0}\right)\left(\mathrm{x}-\mathrm{x}_{1}\right)\left(\mathrm{x}-\mathrm{x}_{2}\right)\left(\mathrm{x}-\mathrm{x}_{3}\right)\left(\mathrm{x}-\mathrm{x}_{4}\right)}{\left(\mathrm{x}_{5}-\mathrm{x}_{0}\right)\left(\mathrm{x}_{5}-\mathrm{x}_{1}\right)\left(\mathrm{x}_{5}-\mathrm{x}_{2}\right)\left(\mathrm{x}_{5}-\mathrm{x}_{3}\right)\left(\mathrm{x}_{5}-\mathrm{x}_{4}\right)} \\
& I_{5}(x)=\frac{(x-302)(x-325)(x-202)(x-201)(x-189)}{(147-302)(147-325)(147-202)(147-201)(147-189)} \\
& =-\frac{1}{3441576600}\left(x^{5}-1219 x^{4}+586103 x^{3}-138992741 x^{2}+16272336156 x-753181310700\right) \\
& P_{5}(x)=l_{0}(x) y_{0}+l_{1}(x) y_{1}+l_{2}(x) y_{2}+l_{3}(x) y_{3}+l_{4}(x) y_{4}+l_{5}(x) y_{5} \\
& P_{5}(x)=\left[\left(-\frac{1}{4068734500}\left(x^{5}-1064 x^{4}+444153 x^{3}-55942774 x^{2}+9227449082 x-368438697900\right)\right) 232\right] \\
& +\left[\left(\frac { 1 } { 8 4 9 2 0 6 9 5 6 8 } \left(x^{5}-1041 x^{4}+426971 x^{3}-86384307 x^{2}+8629369308 x\right.\right.\right. \\
& -340669700532)) 130] \\
& +\left[\left(\frac{1}{8794500}\left(x^{5}-1164 x^{4}+530168 x^{3}-118055946 x^{2}+12856967991 x-548107191450\right)\right) 34\right] \\
& +\left[\left(-\frac{1}{8115552}\left(x^{5}-1165 x^{4}+488045 x^{3}-91377629 x^{2}+8678475432 x-550834092900\right)\right) 49\right] \\
& +\left[\left(\frac { 1 } { 1 0 0 6 9 1 1 3 6 } \left(x^{5}-1177 x^{4}+542843 x^{3}-122552555 x^{2}+13541836188 x\right.\right.\right. \\
& -585807686100)) 151] \\
& +\left[\left(-\frac{1}{3441576600}\left(x^{5}-1219 x^{4}+586103 x^{3}-138992741 x^{2}+16272336156 x\right.\right.\right. \\
& -753181310700)) 18]
\end{aligned}
$$




\subsection{Pante 20 Health Center}

Table 3. A table showing that the number of those who came to Pante 20 Health Center and who were injured

\begin{tabular}{|c|c|c|c|c|c|c|}
\hline Weeks & First week & Second week & Third week & Fourth weak & Fifth week & Sixth week \\
\hline Total came & 227 & 194 & 498 & 1764 & 2904 & 1725 \\
\hline Total patients & 137 & 131 & 348 & 1742 & 2880 & 1688 \\
\hline
\end{tabular}

$$
\begin{aligned}
& \mathrm{l}_{0}(\mathrm{x})=\frac{\left(\mathrm{x}-\mathrm{x}_{1}\right)\left(\mathrm{x}-\mathrm{x}_{2}\right)\left(\mathrm{x}-\mathrm{x}_{3}\right)\left(\mathrm{x}-\mathrm{x}_{4}\right)\left(\mathrm{x}-\mathrm{x}_{5}\right)}{\left(\mathrm{x}_{0}-\mathrm{x}_{1}\right)\left(\mathrm{x}_{0}-\mathrm{x}_{2}\right)\left(\mathrm{x}_{0}-\mathrm{x}_{3}\right)\left(\mathrm{x}_{0}-\mathrm{x}_{4}\right)\left(\mathrm{x}_{0}-\mathrm{x}_{5}\right)} \\
& \mathrm{l}_{0}(\mathrm{x})=\frac{(\mathrm{x}-194)(\mathrm{x}-498)(\mathrm{x}-1764)(\mathrm{x}-2904)(\mathrm{x}-1725)}{(227-194)(227-498)(227-1764)(227-2904)(227-1725)} \\
& =\frac{1}{55121024737086}\left(x^{5}-7085 x^{4}+17695524 x^{3}-18571291668 x^{2}+7387773316272 x-853719821539200\right) \\
& \mathrm{l}_{1}(\mathrm{x})=\frac{\left(\mathrm{x}-\mathrm{x}_{0}\right)\left(\mathrm{x}-\mathrm{x}_{2}\right)\left(\mathrm{x}-\mathrm{x}_{3}\right)\left(\mathrm{x}-\mathrm{x}_{4}\right)\left(\mathrm{x}-\mathrm{x}_{5}\right)}{\left(\mathrm{x}_{1}-\mathrm{x}_{0}\right)\left(\mathrm{x}_{1}-\mathrm{x}_{2}\right)\left(\mathrm{x}_{1}-\mathrm{x}_{3}\right)\left(\mathrm{x}_{1}-\mathrm{x}_{4}\right)\left(\mathrm{x}_{1}-\mathrm{x}_{5}\right)} \\
& \mathrm{l}_{1}(\mathrm{x})=\frac{(\mathrm{x}-227)(\mathrm{x}-498)(\mathrm{x}-1764)(\mathrm{x}-2904)(\mathrm{x}-1725)}{(194-227)(194-498)(194-1764)(194-2904)(194-1725)} \\
& l_{1}(x)=-\frac{1}{65347903262400}\left(x^{5}-7118 x^{4}+17922927 x^{3}-19111127778 x^{2}+7895897735976 x\right. \\
& -998940203553600) \\
& \mathrm{l}_{2}(\mathrm{x})=\frac{\left(\mathrm{x}-\mathrm{x}_{0}\right)\left(\mathrm{x}-\mathrm{x}_{1}\right)\left(\mathrm{x}-\mathrm{x}_{3}\right)\left(\mathrm{x}-\mathrm{x}_{4}\right)\left(\mathrm{x}-\mathrm{x}_{5}\right)}{\left(\mathrm{x}_{2}-\mathrm{x}_{0}\right)\left(\mathrm{x}_{2}-\mathrm{x}_{1}\right)\left(\mathrm{x}_{2}-\mathrm{x}_{3}\right)\left(\mathrm{x}_{2}-\mathrm{x}_{4}\right)\left(\mathrm{x}_{2}-\mathrm{x}_{5}\right)} \\
& \mathrm{l}_{2}(\mathrm{x})=\frac{(\mathrm{x}-227)(\mathrm{x}-194)(\mathrm{x}-1764)(\mathrm{x}-2904)(\mathrm{x}-1725)}{(498-227)(498-194)(498-1764)(498-2904)(498-1725)} \\
& \begin{array}{l}
=-\frac{1}{307905017387387328}\left(x^{5}-6814 x^{4}+15910447 x^{3}-14664773010 x^{2}+4300399565928 x\right. \\
\quad-389145380500800)
\end{array} \\
& \begin{array}{c}
\mathrm{l}_{3}(\mathrm{x})=\frac{\left(\mathrm{x}-\mathrm{x}_{0}\right)\left(\mathrm{x}-\mathrm{x}_{1}\right)\left(\mathrm{x}-\mathrm{x}_{2}\right)\left(\mathrm{x}-\mathrm{x}_{4}\right)\left(\mathrm{x}-\mathrm{x}_{5}\right)}{\left(\mathrm{x}_{3}-\mathrm{x}_{0}\right)\left(\mathrm{x}_{3}-\mathrm{x}_{1}\right)\left(\mathrm{x}_{3}-\mathrm{x}_{2}\right)\left(\mathrm{x}_{3}-\mathrm{x}_{4}\right)\left(\mathrm{x}_{3}-\mathrm{x}_{5}\right)} \\
\mathrm{l}_{3}(\mathrm{x})=\frac{(\mathrm{x}-227)(\mathrm{x}-194)(\mathrm{x}-498)(\mathrm{x}-2904)(\mathrm{x}-1725)}{(1764-227)(1764-194)(1764-498)(1764-2904)(1764-1725)}
\end{array} \\
& =-\frac{1}{135824052452400}\left(x^{5}-5548 x^{4}+9517147 x^{3}-5799928308 x^{2}+1372382989596 x-109860770685600\right) \\
& \mathrm{l}_{4}(\mathrm{x})=\frac{\left(\mathrm{x}-\mathrm{x}_{0}\right)\left(\mathrm{x}-\mathrm{x}_{1}\right)\left(\mathrm{x}-\mathrm{x}_{2}\right)\left(\mathrm{x}-\mathrm{x}_{3}\right)\left(\mathrm{x}-\mathrm{x}_{5}\right)}{\left(\mathrm{x}_{4}-\mathrm{x}_{0}\right)\left(\mathrm{x}_{4}-\mathrm{x}_{1}\right)\left(\mathrm{x}_{4}-\mathrm{x}_{2}\right)\left(\mathrm{x}_{4}-\mathrm{x}_{3}\right)\left(\mathrm{x}_{4}-\mathrm{x}_{5}\right)} \\
& \mathrm{l}_{4}(\mathrm{x})=\frac{(\mathrm{x}-227)(\mathrm{x}-194)(\mathrm{x}-498)(\mathrm{x}-1764)(\mathrm{x}-1725)}{(2904-227)(2904-194)(2904-498)(2904-1764)(2904-1725)} \\
& =\frac{1}{23460212495041200}\left(x^{5}-4408 x^{4}+6502987 x^{3}-3703501365 x^{2}+848488552236 x-585807686100\right) \\
& \mathrm{l}_{5}(\mathrm{x})=\frac{\left(\mathrm{x}-\mathrm{x}_{0}\right)\left(\mathrm{x}-\mathrm{x}_{1}\right)\left(\mathrm{x}-\mathrm{x}_{2}\right)\left(\mathrm{x}-\mathrm{x}_{3}\right)\left(\mathrm{x}-\mathrm{x}_{4}\right)}{\left(\mathrm{x}_{5}-\mathrm{x}_{0}\right)\left(\mathrm{x}_{5}-\mathrm{x}_{1}\right)\left(\mathrm{x}_{5}-\mathrm{x}_{2}\right)\left(\mathrm{x}_{5}-\mathrm{x}_{3}\right)\left(\mathrm{x}_{5}-\mathrm{x}_{4}\right)} \\
& \mathrm{l}_{5}(\mathrm{x})=\frac{(\mathrm{x}-227)(\mathrm{x}-194)(\mathrm{x}-498)(\mathrm{x}-1764)(\mathrm{x}-2904)}{(1725-227)(1725-194)(1725-498)(1725-1764)(1725-2904)} \\
& =-\frac{1}{129392760675906}\left(x^{5}-5587 x^{4}+9666244 x^{3}-5913904716 x^{2}+1401970889808 x-112344579414144\right)
\end{aligned}
$$




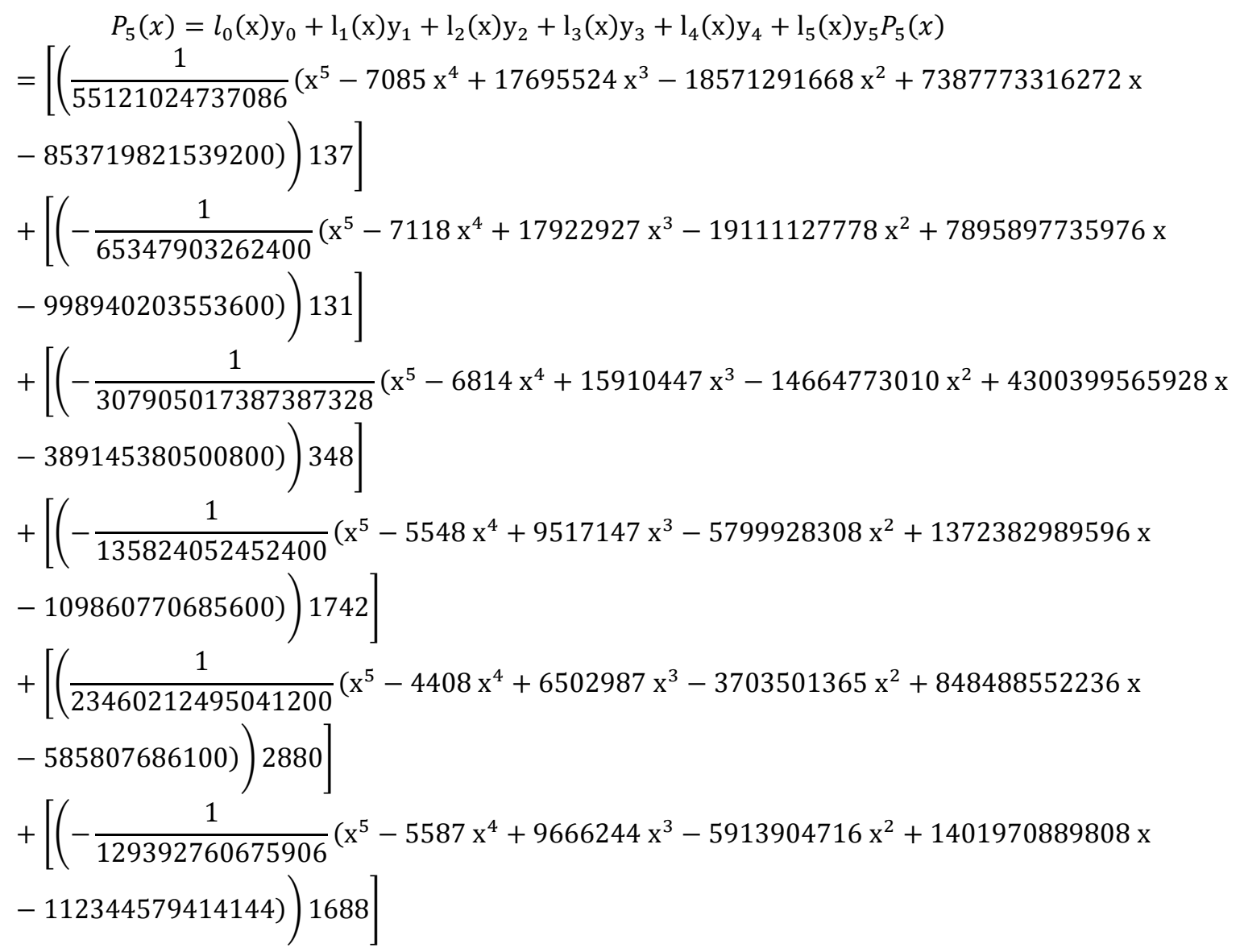

\section{Results and Discussion}

\subsection{Rail Way Health Center}

Table 4. A table showing that the mathematical treatment using Matlab for the number of those who came to Railway Health Center and who were injured

\begin{tabular}{|c|c|c|c|c|c|c|}
\hline Weeks & First week & Second weak & Third week & Fourth week & Fifth week & Sixth week \\
\hline Total came & 208 & 395 & 389 & 258 & 256 & 120 \\
\hline Total patients & 113 & 266 & 253 & 194 & 116 & 32 \\
\hline
\end{tabular}

$$
P_{5}(x)=l_{0}(\mathrm{x}) \mathrm{y}_{0}+\mathrm{l}_{1}(\mathrm{x}) \mathrm{y}_{1}+\mathrm{l}_{2}(\mathrm{x}) \mathrm{y}_{2}+\mathrm{l}_{3}(\mathrm{x}) \mathrm{y}_{3}+\mathrm{l}_{4}(\mathrm{x}) \mathrm{y}_{4}+\mathrm{l}_{5}(\mathrm{x}) \mathrm{y}_{5}
$$

Solution

function $[1, \mathrm{~L}]=\operatorname{lagranp}(\mathrm{x}, \mathrm{y})$

\%Input: $\mathrm{x}=[\mathrm{x} 0 \mathrm{x} 1 \ldots \mathrm{xN}], \mathrm{y}=[\mathrm{y} 0 \mathrm{y} 1 \ldots \mathrm{yN}]$

\%Output: 1 = Lagrange polynomial coefficients of degree $\mathrm{N}$

$\% \mathrm{~L}=$ Lagrange coefficient polynomial

$\mathrm{N}=$ length$(\mathrm{x})-1 ; \%$ the degree of polynomial

$1=0$;

for $\mathrm{m}=1: \mathrm{N}+1$

$\mathrm{P}=1$;

for $\mathrm{k}=1: \mathrm{N}+1$

if $\mathrm{k} \sim=\mathrm{m}, \mathrm{P}=\operatorname{conv}(\mathrm{P},[1-\mathrm{x}(\mathrm{k})]) /(\mathrm{x}(\mathrm{m})-\mathrm{x}(\mathrm{k}))$; end end

$\mathrm{L}(\mathrm{m},:)=\mathrm{P} ; \%$ Lagrange coefficient polynomial

$1=1+y(m) * P$

end

$\operatorname{plot}\left(\mathrm{x}, \mathrm{y}, \mathrm{r}^{\prime}\right)$

xlabel('Total came')
ylabel('Total patients')

title('Rail way health center')

gridon

\%do_lagranp.m

$\mathrm{x}=\overline{[2} 08395389258256120] ; \mathrm{y}=\left[\begin{array}{lll}113 & 266253194116\end{array}\right.$

32]; \% given data points

$1=$ lagranp $(\mathrm{x}, \mathrm{y}) \%$ find the Lagrange polynomial

$\mathrm{xx}=[-2: 0.02: 2] ; \mathrm{yy}=\operatorname{polyval}(1, \mathrm{xx}) ; \%$ interpolate for $[-$ 2,2]

clf, plot(xx,yy,'b', x,y,'*') \%plot the graph

Result:

$>>$ Lagrange $(x, y)$

ans $=$

$1.0 \mathrm{e}+05 *$

0.0000-0.00000.0000-0.00060.0684-3.0888

Plotting of Result: 


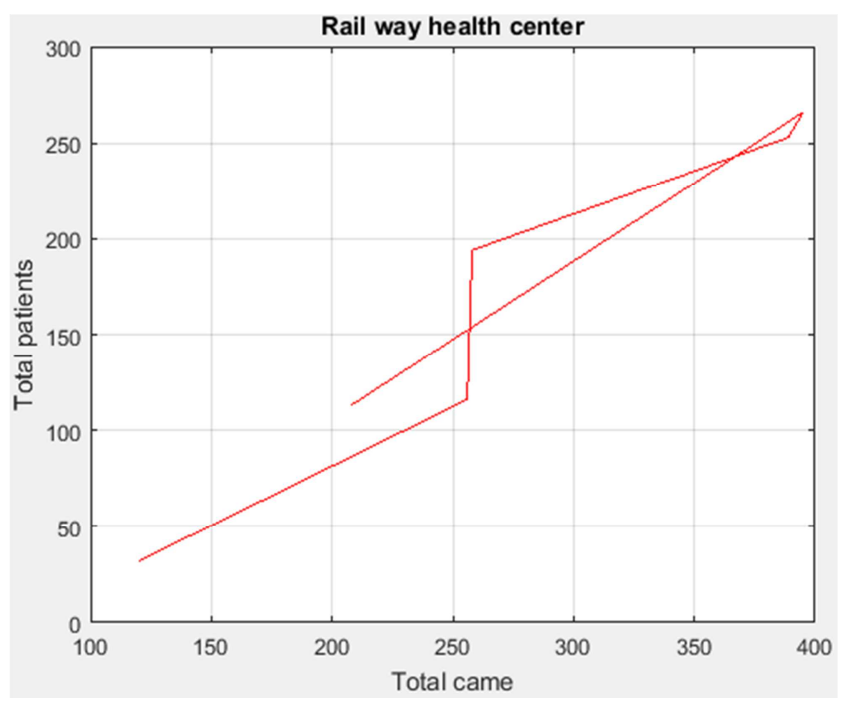

Figure 1. Instability of patients cases.

\subsection{Pante 42 Health Center}

Table 5. A table showing that the mathematical treatment using Matlab for the number of those who came to Pante 42 Health Center and who were injured.

\begin{tabular}{lllllll}
\hline weeks & $\begin{array}{l}\text { First } \\
\text { week }\end{array}$ & $\begin{array}{l}\text { Second } \\
\text { week }\end{array}$ & $\begin{array}{l}\text { Third } \\
\text { weak }\end{array}$ & $\begin{array}{l}\text { Four } \\
\text { weak }\end{array}$ & $\begin{array}{l}\text { Five } \\
\text { weak }\end{array}$ & $\begin{array}{l}\text { Six } \\
\text { week }\end{array}$ \\
\hline Total came & 302 & 325 & 202 & 201 & 189 & 147 \\
Total patients & 232 & 130 & 34 & 49 & 151 & 18 \\
\hline
\end{tabular}

Solution

function $[1, \mathrm{~L}]=\operatorname{lagranp}(\mathrm{x}, \mathrm{y})$

\%Input: $\mathrm{x}=[\mathrm{x} 0 \mathrm{x} 1 \ldots \mathrm{xN}], \mathrm{y}=[\mathrm{y} 0 \mathrm{y} 1 \ldots \mathrm{yN}]$

$\mathrm{N}$

$\%$ Output: $1=$ Lagrange polynomial coefficients of degree

$\% \mathrm{~L}=$ Lagrange coefficient polynomial

$\mathrm{N}=$ length(x)-1; \%the degree of polynomial

$1=0$;

for $\mathrm{m}=1: \mathrm{N}+1$

$\mathrm{P}=1$;

for $\mathrm{k}=1: \mathrm{N}+1$

if $\mathrm{k} \sim=\mathrm{m}, \mathrm{P}=\operatorname{conv}(\mathrm{P},[1-\mathrm{x}(\mathrm{k})]) /(\mathrm{x}(\mathrm{m})-\mathrm{x}(\mathrm{k}))$; end end

$\mathrm{L}(\mathrm{m},:)=\mathrm{P} ; \%$ Lagrange coefficient polynomial

$1=1+\mathrm{y}(\mathrm{m}) * \mathrm{P}$

end

$\operatorname{plot}\left(\mathrm{x}, \mathrm{y}, \mathrm{r}^{\prime}\right)$

xlabel('Total came')

ylabel('Total patients')

title('Rail way health center')

gridon

\%do lagranp.m

$\mathrm{x}=\left[\begin{array}{llllll}302 & 325 & 202 & 201 & 189 & 147\end{array}\right] ; \mathrm{y}=\left[\begin{array}{lllll}232 & 130 & 34 & 49 & 151\end{array}\right.$

18]; \% given data points

$1=$ lagranp $(\mathrm{x}, \mathrm{y}) \%$ find the Lagrange polynomial

$\mathrm{xx}=[-2: 0.02: 2] ; \mathrm{yy}=\operatorname{polyval}(1, \mathrm{xx}) ; \%$ interpolate for $[-$ $2,2]$

clf, plot(xx,yy,'b', x,y,'*') \%plot the graph

Result: ans $=$

$1.0 \mathrm{e}+05 *$

$-0.00000 .0000-0.00000 .0008-0.08403 .4794$

Plotting of Result:

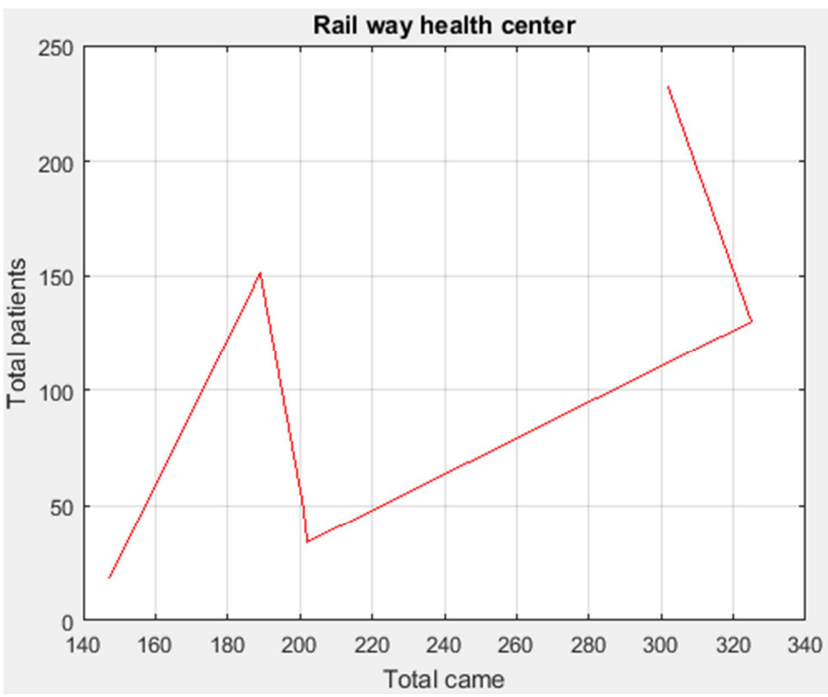

Figure 2. Unnatural Geometric Interpretation using Matlab for the number of those who came to Railway Health Center and who were injured

\subsection{Pante 20 Health Center}

Table 6. A table showing that the mathematical treatment using Matlab for the number of those who came to Pante 20 Health Center and who were injured.

\begin{tabular}{lllllll}
\hline Week & $\begin{array}{l}\text { First } \\
\text { week }\end{array}$ & $\begin{array}{l}\text { Second } \\
\text { week }\end{array}$ & $\begin{array}{l}\text { Third } \\
\text { week }\end{array}$ & $\begin{array}{l}\text { Fourth } \\
\text { weak }\end{array}$ & $\begin{array}{l}\text { Fifth } \\
\text { week }\end{array}$ & $\begin{array}{l}\text { Sixth } \\
\text { week }\end{array}$ \\
\hline Total came & 227 & 194 & 498 & 1764 & 2904 & 1725 \\
Total patients & 137 & 131 & 348 & 1742 & 2880 & 1688 \\
\hline
\end{tabular}

Solution

function $[1, \mathrm{~L}]=\operatorname{lagranp}(\mathrm{x}, \mathrm{y})$

\%Input: $\mathrm{x}=[\mathrm{x} 0 \mathrm{x} 1 \ldots \mathrm{xN}], \mathrm{y}=[\mathrm{y} 0 \mathrm{y} 1 \ldots \mathrm{yN}]$

\%Output: 1 = Lagrange polynomial coefficients of degree

$\mathrm{N}$

$\% \mathrm{~L}=$ Lagrange coefficient polynomial

$\mathrm{N}=$ length$(\mathrm{x})-1 ; \%$ the degree of polynomial

$1=0$;

for $\mathrm{m}=1: \mathrm{N}+1$

$\mathrm{P}=1$;

for $\mathrm{k}=1: \mathrm{N}+1$

if $\mathrm{k} \sim=\mathrm{m}, \mathrm{P}=\operatorname{conv}(\mathrm{P},[1-\mathrm{x}(\mathrm{k})]) /(\mathrm{x}(\mathrm{m})-\mathrm{x}(\mathrm{k}))$; end

end

$\mathrm{L}(\mathrm{m},:)=\mathrm{P} ; \%$ Lagrange coefficient polynomial

$\mathrm{l}=1+\mathrm{y}(\mathrm{m}) * \mathrm{P}$;

end

plot(x,y,'r')

xlabel('Total came')

ylabel('Total patients')

title('Rail way health center')

gridon

\%do lagranp.m

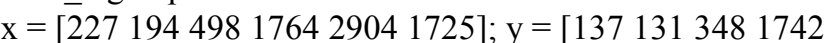

2880 1688]; \% given data points 
$1=$ lagranp $(\mathrm{x}, \mathrm{y}) \%$ find the Lagrange polynomial $\mathrm{xx}=[-2: 0.02: 2] ; \mathrm{yy}=\operatorname{polyval}(1, \mathrm{xx}) ; \%$ interpolate for $[-2,2]$ clf, plot(xx,yy,'b', x,y,'*') \%plot the graph

Result:

$>>$ Lagrange $(\mathrm{x}, \mathrm{y})$

ans $=$

$-0.00000 .0000-0.00000 .0055-1.5348255 .7012$

Plotting of Result:

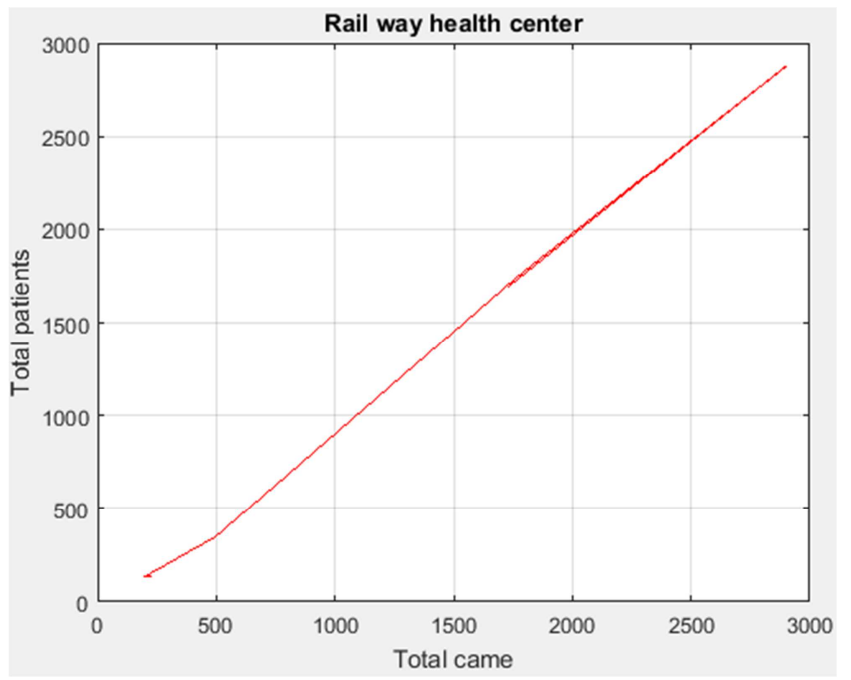

Figure 3. Natural Geometric Interpretation using Matlab for the number of those who came to Railway Health Center and who were injured.

\section{Compare Between Numerical and Proposed Modeling}

Table 7. Comparison between Numerical and Matlab solutions.

\begin{tabular}{lll}
\hline & Numerical Modeling & Proposed Modeling \\
\hline Accurate & Non accurate & Accurate \\
fast & Not Fast & Fast \\
MultiValues & Non-Available & Available \\
General Results & Non Quality & Quality \\
\hline
\end{tabular}

\section{Conclusion}

Finally we note that from table 1 and table 2 Chikungunya disease start decreasing in third week but in table 3 we found that Chikungunya disease decreasing in sixth week also we explained the possibility of the modeling of Chikungunya disease using the Lagrange interpolating method by Matlab.

\section{References}

[1] Braiala Wahida, AmjadAli, Shazia Rafique, Muhammad Idrees, Global expansion of chikungunya virus:mapping the 64-yearhistory, International Journal of Infectious Diseases, Corresponding Editor: Eskild Petersen, Aarhus, Denmark, March 2017.

[2] Claudio Soto-Garita MSc Candidate, Jean-paul Carrera MSc, Sandra Lopez-verges Phd \& Eugenia Corrales-Aguilar Advances in Clinical Diagnosis and Management of
Chikungunya Virus infection Neglected Tropical diseases (A Sanchez, Section Editor)Published: 08 May 2018.

[3] Dr. Margaret Chan, Director-General, World Health Organization, World Health Day 2014 - Vector-borne diseases.

[4] LF da Rochajr, HD delima, RM Correia, MRDA freitas, PRS de melo, AGL de Mattos, AFR de Olivera, CDL Marques, ALBP Duarte, O Lins, A Ranzolin, SAT0566 Electroneuro graphic findings in patients with subacute/chronic articular symptoms of chikungunya fever and neuropathic complaints preliminary results, Copyright information:(C) 2017, Published by the BMJ Publishing Group Limited.

[5] M. Runowska, D. Majewski, K. Niklas, M. Puszczewicz, Chikungunya virus: a rheumatologist's perspective, Clinical and Experimental Rheumatology 2018.

[6] Meghna R. Sebastian, RakeshLodha, and S. K. Kabra, Chikungunya Infection in Children, Indian Journal of Pediatrics, Volume 76-February 2009.

[7] Md. Tanvir Rahman, Chikungunya virus infection in developing countries - What should we do?, Journal of Advanced Veterinary and Animal Research, ISSN 2311-7710 (Electronic), Vol 4 No 2, Pages 125-131. June 2017.

[8] Muktha S. Natrajan, Alejandra Rojas, Jesse J. Waggoner, Beyond Fever and Pain: Diagnostic Methods for Chikungunya Virus, Copyright $\subset 2019$ American Society for Microbiology.

[9] Nazish Badar, Muhammad Salman, Jamil Ansari, Uzma Aamir, Muhammad MasroorAlam, Yasir Arshad, NighatMushtaq, Aamer Ikram, Javaria Qazi, Emergence of Chikungunya Virus, Pakistan, 2016-2017, Emerging Infectious Diseases • www.cdc.gov/eid • Vol. 26, No. 2, February 2020.

[10] Nildimar Alves Honório, Keenan Wiggins, Bradley Eastmond, Experimental Vertical Transmission of Chikungunya Virus by Brazilian and Florida, 2019.

[11] Nyazi Tawfeeg Ahmed - Numerical Analysis proBm-lems Sudan university of Science and Technology college of Engineering, 2001.

[12] Paul F Hord, Philippe buchy, Chikungunya, Article (PDF Available) in Revue scientifique et technique (International Office of Epizootics) 34 (2): 479-489 · August 2015 with 6,518 Reads.

[13] Pan American World Health Organization, World Health Organization, 2014.

[14] Pan American World Health Organization, World Health Organization Information for Health care Providers Chikungunya Fever January 2014 CHICKUNGUNYA.

[15] Samlee Plianbangchang, M. D., Dr. P. H. Regional Director, Guidelines on Clinical Management of Chikungunya Fever, World Health Organization 2008, Printed in India.

[16] Sudanese Federal Ministry of Health, October 2018.

[17] World Health Organization, Regional Office for South-East Asia, ISBN 978-92-9022-337-5, Printed in India, 2009.

[18] World Health Organization, Guidelines on Clinical Management of Chikungunya Fever, Printed in India, October 2008. 